\title{
Article
}

\section{The impact of wrong-site surgery on dental undergraduate teaching: a survey of UK dental schools}

Dargue, Anna, Fyfe, Eithne, French, Kathryn, Ali, Kamran, Bailey, Edmund, Bell, Aileen, Bolt, Robert, Bulsara, Yogesh, Carey, James, Emanuel, Charlotte, Green, Rachel, Khawaja, Nadine, Kushnerev, Evgeny, Patel, Neil, Shepherd, Simon, Smart, Binthan, Smyth, Joanna, Taylor, Kate and Datla, Kumar Varma

Available at http://clok.uclan.ac.uk/36237/

Dargue, Anna, Fyfe, Eithne, French, Kathryn, Ali, Kamran, Bailey, Edmund, Bell, Aileen, Bolt, Robert, Bulsara, Yogesh, Carey, James et al (2021) The impact of wrong-site surgery on dental undergraduate teaching: a survey of UK dental schools. European Journal of Dental Education, 25 (4). pp. 670-678. ISSN 1396-5883

It is advisable to refer to the publisher's version if you intend to cite from the work. http://dx.doi.org/10.1111/eje.12645

For more information about UCLan's research in this area go to http://www.uclan.ac.uk/researchgroups/ and search for <name of research Group>.

For information about Research generally at UCLan please go to http://www.uclan.ac.uk/research/

All outputs in CLoK are protected by Intellectual Property Rights law, including Copyright law. Copyright, IPR and Moral Rights for the works on this site are retained by the individual authors and/or other copyright owners. Terms and conditions for use of this material are defined in the policies page.

\section{CLoK}

Central Lancashire online Knowledge www.clok.uclan.ac.uk

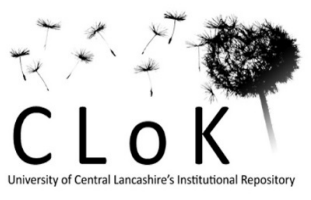


The impact of wrong-site surgery on dental undergraduate teaching: a survey of UK dental schools

\section{ABSTRACT}

Introduction: Patient safety within dental education is paramount. Wrong site surgery (WSS) tooth extraction is not uncommon and is a significant Never Event (NE) in dentistry. This study aims to explore dental schools' undergraduate experience of NEs, safety interventions implemented and the impact on student experience.

Methods: All 16 UK Dental Schools were surveyed via e-mail.

Results: The response rate was $100 \%$. A modified WHO checklist was used within institutions (94\%) including pre-operative briefings and recording teeth on whiteboards (81\% respectively). Students were directly supervised performing extractions $(63 \%)$ utilising a 1:4 Staff: Student ratio. WSS by students was reported in $69 \%$ of schools, with student experience being impacted by an increased patient safety focus.

Discussion: This study demonstrated an increased utilisation of an adapted WHO checklist. Modification of practices to ensure patient safety was demonstrated at all schools, irrespective of student WSS occurrences. Institutions experiencing student NEs commonly implemented WHO checklists and recording teeth for extraction on whiteboards. Other strategies included direct staff supervision and pre-operative briefings.

Conclusion: UK Dental Schools have increased the emphasis on patient safety by the implementation of national healthcare models e.g. WHO checklists and preoperative briefings. These strategies both aim to improve communication and teamwork. Increased levels of staff supervision foster greater quality of teaching however, this has resulted in reduced student clinical experience. A proposed minimum standard for undergraduate surgery is suggested to ensure safe and competent dental practitioners of the future.

Keywords: never event, oral surgery, patient safety, WHO checklist, wrong site surgery, undergraduate 


\section{Introduction}

Patient safety in healthcare has come under sharper focus over the last twenty years. The landmark report by the Institute of Medicine in 1999 "To Err is Human: Building a Safer Health System" documented that up to 98,000 inpatient deaths per year within the USA were the result of medical errors. ${ }^{1}$ United Kingdom medical communities have not been immune to reports highlighting poor standards of care and avoidable harmful events. The Francis report, published following the public inquiry into the failings at Mid-Staffordshire Hospital, has had ramifications which included the Berwick review of patient safety within the NHS. ${ }^{2,3}$

Greater emphasis on patient safety has been mandated within dental education. The General Dental Council's (GDC) 2015 documents "Preparing for Practice" and "Standards for Education" highlight the importance of safety and quality of care for patients, and in particular the documents stress that providers of dental education must ensure that patient safety is paramount. 4,5 Similarly, the Association of Dental Education Europe (ADEE) updated its curriculum in 2017 with increased prominence given to patient safety and an expectation that its curriculum would enhance this. ${ }^{6}$

Strategies to foster strong patient safety cultures have been advised as a way to minimise errors in healthcare. ${ }^{7}$ In the USA, researchers noted a more positive patient safety culture within dental schools when compared to medical hospitals, although weaknesses in reporting events and organisational learning were identified in the school setting. ${ }^{8}$ A recent study of a UK Dental School identified that students had a good understanding and positive attitude towards patient safety. ${ }^{9}$

In 2009, the UK National Patient Safety Agency (NPSA) introduced the concept of "Never Events" (NE), defined as 'serious patient safety incidents that are wholly preventable because guidance or safety recommendations that provide strong systemic protective barriers are available at a national level and should have been implemented by all healthcare providers.' ${ }^{10}$ Wrong tooth extraction, was included under wrong-site surgery (WSS) in April 2015 and is the most frequently reported NE relevant to dentistry. ${ }^{11}$ Table 1 presents the number of reported wrong tooth extractions and wrong-site surgeries per year in England highlighting the significance of operator error. ${ }^{12}$ All commissioners and providers of NHS care (public healthcare) in England are required to report NEs. The majority of these figures correspond to secondary care settings such as hospitals trusts, as under-reporting in primary care 
dentistry is likely due to complex reporting systems. ${ }^{13}$ Between 2016 and 2019 wrong tooth extraction was the most common WSS reported.

Analysis of wrong tooth extraction data suggests that cognitive failure is one of the most frequent causes, alongside failures in communication and training. ${ }^{14,15}$ In addition it is reasonable to assume that the common causes cited in relation to the errors of medical trainees, namely lack of supervision and lack of technical skills, would also relate to dental students. ${ }^{16,17}$ In 2016 a survey of all UK and Irish Dental Hospitals identified that the majority of the Dental Schools were using surgical safety checklists for outpatient extractions. ${ }^{18}$

The aim of our survey was to explore the UK Dental Schools' experiences of undergraduate WSS tooth extractions, what interventions had been implemented following root cause analysis to prevent recurrence, and to understand the impact of these on student experience. The Berwick report emphasised the importance of the NHS embracing transparency and openness and the need to become a learning organisation. ${ }^{3}$ It is our hope that by sharing our teaching hospitals' experiences, this goes some way to embracing transparency. 


\section{Methods}

An initial draft document was sent by email to all sixteen dental schools' representatives from the education committee of the Association of British Academic Oral and Maxillofacial Surgeons (ABAOMS). Modifications were made to the survey using an informal Delphi approach to generate expert consensus. The final version was a self-completed questionnaire consisting mostly of closed questions with space for additional comments. The final survey (Appendix 1) was sent electronically to all sixteen undergraduate dental schools in the United Kingdom in the winter of 2019. One reminder was sent by email after a month had elapsed.

Consideration of the need for formal ethical approval was made by the principal organisers ( $A D, E F$ and $K F)$, however this was a collaborative survey with mutual benefit for all the clinicians and educators involved. The aim was to share the findings with both the education committee and the wider dental education community. Ethical approval was deemed unnecessary as the survey included non-sensitive questions, participants were not considered to be "vulnerable" and the survey was not deemed to cause any detrimental impact. ${ }^{19}$ 


\section{Results}

All sixteen Dental Schools in the United Kingdom completed the survey. Ninety four percent of schools used a modified World Health Organisation (WHO) checklist for extractions carried out under local anaesthesia by undergraduate dental students. Seven schools (44\%), introduced a WHO checklist for undergraduate treatment in the period up to and including 2014. These schools were early adopters of the WHO checklist, prior to wrong tooth extraction being defined as a NE under the WSS theme. A further eight schools (50\%) had introduced the use of a WHO checklist between 2015 and 2019. Only one school was not using a WHO checklist, this was due to an apparent lack of engagement by relevant stakeholders. The majority of dental schools $(81 \%)$ also performed pre-operative safety briefings with undergraduate students prior to treatment sessions commencing.

Additional pre-operative checks, namely a whiteboard to record those teeth to be extracted, were utilised (81\%). Three of these schools used further supplementary checks: two schools recorded the same information on the patient's bib, one school physically marked the tooth with red wax or, if a root was to be removed, used a skin marker on the gingivae. An additional safety precaution implemented by these three schools included clinical staff directly supervising students throughout treatment. Of the three schools (19\%) that did not use a whiteboard, one school instead marked radiographs as a further step to preventing WSS. Furthermore, these three schools ensured staff directly supervised the students throughout the entire course of the extraction.

Interestingly there was a variable level of student supervision during the extraction procedure (Figure 1), some of which was due to staffing levels and others based on the level of competence of the student. The majority of schools $(63 \%)$, ensured clinical staff were present throughout student extractions, regardless of student seniority. Three schools used both clinical and nursing staff at all times. The remaining five schools (31\%) had a minimum requirement that students confirm with staff that the forceps were on the correct tooth before being allowed to commence the extraction. In two of these five schools, after confirmation staff continued to directly supervise junior students throughout the procedure. Three respondents commented about departmental minimum standards of supervision and detailed that their aim was to supervise each student as much as possible. A number of external factors affected 
patient flow e.g. individual patient factors, difficulty of extraction, lack of student ability, time pressures and staff shortages.

Significant variation in student numbers per year and staff: student ratios were identified (Table 2). Student cohort sizes ranged between 60 to 80 and 80 to 100 students (63\%: $25 \%$ respectively). The average ratio of staff to students was one staff member supervising four students (50\% of schools) with $38 \%$ of schools having a ratio of 1:3. Further data mining identified that the Dental School with the least number of students (30) had the highest ratio of staff supervision (1:1).

The number of WSS tooth extractions by undergraduate students since 2009 was investigated (Figure 2). A total of 23 wrong tooth extractions were recorded as occurring in the decade 2009-2019 by undergraduates in UK dental schools. (The other wrong tooth extractions thus occurred in other NHS care settings such as district general hospitals, primary care, community or were carried out by qualified staff in UK dental schools, see Table 1.) Approximately $70 \%$ of dental teaching hospitals had experienced students removing the incorrect tooth, with one school reporting five NEs over this period. Five schools $(31 \%)$ reported no episodes of wrong tooth extraction.

Schools with experience of WSS by undergraduates $(69 \%)$ were asked followup questions regarding which interventions had been implemented to prevent these occurring again. The majority of schools (nine of eleven schools) had introduced the use of a modified WHO checklist and used a whiteboard to record teeth as additional pre-operative checks in order to prevent a recurrence of wrong tooth extractions. A further five schools instigated pre-operative briefings with dental students. Dental schools increased direct staff supervision by either clinical staff or nursing staff $(6,2$ schools respectively). Less frequently, three schools used the patient's bib to record which teeth were being removed, and two schools had partnered students together. Some respondents provided additional comments that the patient safety changes made in their departments did not just relate to student experience of WSS, these were also made in relation to staff events and in response to the increasing national focus on patient safety.

This study also identified the impact of WSS protocols and the increasing focus on patient safety on student experience. Comments made by each dental school were summarised according to the overall theme of the effect on student experience as: positive, negative, mixed or no effect. Percentages were then calculated for each effect. Overall most felt there was a mixed effect on student experience $(56 \%)$, whilst 
$31 \%$ identified a positive effect and $6 \%$ a negative effect or no effect. Table 3 presents the detailed results for agreement with statements each school made, with some schools making several statements about the impact on student experience. Analysis of these themes identified potential causes for these opinions. For the majority of respondents, a formalised process of checks and students being more aware of the importance of patient safety were identified as notable benefits. However, the lack of student independence in treating patients was seen to deliver a significant adverse impact by many, as well as the increased burden of paperwork resulting in students seeing fewer patients. 


\section{Discussion}

It is very encouraging that all UK Dental Schools took part in this study in a spirit of openness, exploring dental schools' experience of undergraduate NEs and the measures that had been implemented to prevent these from recurring. The results of this survey go some way to understanding student, staff and institutional engagement with patient safety measures.

WHO checklists are now an almost universal part of the undergraduate oral surgery experience. A number of authors from teaching hospitals have described their proactive implementation of modified WHO checklists within the outpatient setting and the importance of a student-led checklist that encourages a patient-safety mindset. ${ }^{20,21}$ Given a systematic review elucidated that surgical safety checklists are the only intervention in dentistry that reduce or minimise adverse events, adoption of the WHO checklist during undergraduate teaching sessions, is vital. ${ }^{22}$ A 2015 survey of all UK and Irish Dental Hospitals found that $77 \%$ of schools were using a surgical safety checklist to prevent wrong tooth extraction in an outpatient setting. ${ }^{18}$ It is reassuring that this improvement is continuing, with our survey now showing current adoption in all schools but one. One school reported that a checklist had not been implemented due to a lack of engagement by relevant stakeholders. Resistance by senior staff is a recognised barrier to successful implementation. ${ }^{23}$

Most Dental Schools employed pre-operative safety briefings with undergraduate students prior to treatment sessions. A paper describing adoption of a pre-operative 'huddle' within an oral surgery department found that qualified staff felt

it improved teamwork, communication and improved overall patient safety. ${ }^{24}$ Specific to dental undergraduates, a survey of dental students in their fourth year of study noted that they already valued pre-procedure briefings for safe and effective clinical practice prior to a patient safety educational module. ${ }^{25} \mathrm{NHS}$ England also advocates the use of such briefings. ${ }^{23}$

The most common causes of WSS tooth extraction are recognised as cognitive failure and miscommunication. ${ }^{14,15}$ Additional safety checks adopted were used to improve communication and provide barriers to cognitive failure, the most frequent being recording the teeth to be extracted on a whiteboard. Most schools use this technique, and for the majority, this had been introduced in response to undergraduate WSS. Saksena et al found their staff valued whiteboards as a communication and verification tool and saw it as integral to safety processes. ${ }^{20}$ 
Dentistry is a unique undergraduate course where students undertake surgery on patients prior to qualification. Given that oral surgery has been noted to have some of the most serious and irreversible complications in dentistry, there is a real potential for dental students to cause patient harm. ${ }^{26}$ Studies have highlighted lack of technical skills and lack of supervision as leading causes of medical trainee error whilst patient outcomes have been observed to improve with direct staff supervision. ${ }^{16,17,27}$ It is therefore unsurprising that the majority of dental schools ensure staff directly supervise students throughout the entire procedure. Fifty percent of schools had introduced continuous supervision of students in response to a WSS tooth extraction within their department, representing a significant change in teaching to prevent future error. The average staff/student ratio for supervision of routine extractions is 1:4 (range $1: 1-1: 6)$. An improvement in levels of staff supervision has been observed when compared to a survey in 2006, where the average ratio was $1: 5 .{ }^{26}$ The increased national focus on patient safety and resultant emphasis within dental education are likely to account for the increase in staff supervision of undergraduates.

Most schools had experience of WSS by an undergraduate in the past ten years. An examination of data for the quantity and ratio of staff supervision, student numbers and types of pre-operative checks performed in schools that had experienced a NE revealed no common associations. The survey responses are deemed representative as there was a $100 \%$ return rate and the largest sample size possible as all UK dental schools were involved. Of note from our survey, those schools reporting no experience of NEs were still proactive in improving patient safety as part of quality improvement and educational requirements. Respondents noted staff NEs and the national impetus to prevent medical errors as prompts to implement enhanced patient safety measures.

The impact of patient safety interventions on the undergraduate student experience was varied with the most commonly cited benefits being a formalised process for safety checks and students having greater awareness of patient safety. However, one third of staff felt students did not understand the significance of these checks. This variable view by staff of student understanding of patient safety is at odds with a recent survey of UK dental students that found students had a positive attitude and sound understanding of patient safety. ${ }^{9}$ In our survey, one third of the teaching staff stated that patient experience was improved due to increased staff supervision of undergraduates: this has been demonstrated to improve patient 
outcomes. ${ }^{27}$ Increased supervision also resulted in the higher quality teaching that was noted as a positive change by a quarter of academic staff. The most frequently cited negative impacts of patient safety interventions were lack of opportunity for student independence in treating patients and additional paperwork. Half of teaching staff felt students had less clinical experience. The question remains whether the perceived positives of a better patient experience and higher quality teaching offset the perceived disadvantages of delivering less clinical experience and little experience of working autonomously. This survey has brought to light some uncertainty around this balance.

With most schools having a supervision ratio of $1: 4$ and an average of 80 students per year, it is a significant challenge to balance patient safety with sufficient clinical experience; as has been highlighted previously. ${ }^{26}$ The increasing patient safety requirements add an additional layer of complexity and pressure on teaching staff. Survey respondents underscored their aim to give students as much supervision and support as possible when performing extractions, however they also described the conflicting demands of difficulty of extraction, lack of student ability, patient factors and time pressures hampering these efforts. Shortages of academic staff further compound these problems. ${ }^{26}$

The significant benefit of national recognition of NEs is to provide a focus to review safety culture, policies and practice. ${ }^{18}$ Encouragingly, all schools that had experienced NEs caused by undergraduates had implemented changes to improve patient safety. A move towards a more just culture of analysing systems rather than placing blame on the practitioner is vital to effecting positive change. ${ }^{7}$ Failing to report errors and share learning have previously been identified as weaknesses in dental schools when compared to hospitals. ${ }^{8}$ However, our survey highlights many dental schools have shown a proactive approach to patient safety.

This survey provides valuable oral surgery centred data on patient safety incidences within dental schools, shares learning on a national level and now proposes a minimum safety standard for undergraduate dental students performing extractions (Figure 3). This profession-led proposal for standardising procedures and embedding best practice fulfils NHS England's strategy to foster the conditions for safer surgery. ${ }^{23}$ As a group, the ABAOMS education committee highlight that the patient safety changes that have been implemented in UK Dental Hospitals have been a highly positive step with the creation of a 'patient safety culture' in dental schools. Now is the time to ensure adequate staffing of these units, to ensure that students view this 
practice as the 'normal' standard of care given. ${ }^{9}$ As already emphasised, dentistry is a unique undergraduate course where there is the potential for patient harm as students develop their skills. This survey however, also highlights the reduced student experience and fewer possibilities for autonomous practice due to the increasing levels of staff supervision as a result of an increased patient safety focus. It is important to acknowledge that dental education is a continuum from the limited clinical experience of newly qualified graduates and continues with mentorship during foundation training with a focus to become proficient in procedural skills. ${ }^{28,29}$ However, it must be remembered that a dental graduate lacking confidence and experience is more likely to refer patients to secondary care drawing further on precious NHS resources. ${ }^{30}$ The outcomes of the undergraduate oral surgery curriculum are to deliver new graduates with the ability to work independently in primary care. ${ }^{31}$

Whilst there is no evidence for fewer incidences of NEs with increased staff supervision, it is notable that half of the teaching staff surveyed felt that students had reduced clinical experience due to the administrative burden and longer waiting time for supervision. Half of all UK dental schools already have a ratio of 1:4 staff to students and over a third of dental schools have 1:3, one further school has an enviable 1:1 supervision. It stands to reason that increased levels of staff supervision, when students are undertaking irreversible treatment on patients, is a constructive goal. It is our suggestion, based on the collective expert opinion of teaching staff that schools should be aiming for a minimum supervision ratio of 1:3. This allows for appropriate teaching input in a positive patient safety environment, with sufficient clinical experience for student development. Staff should, however, be allowed local discretion for competent students to develop their autonomous practice and confidence in their ability to perform procedures independently, within a safe operating setting. Bloom's mastery learning model, where instruction varies according to the needs of the student, would support this technique and has been shown to be an effective supervisory method according to postgraduate dental students. ${ }^{32,33}$ Allowing students the opportunity to experience independent practice within the confines of a more supportive dental school environment rather than a sudden transition in their foundation training year will be of benefit during this time of rapid learning. These aims must be set against the backdrop of challenges recruiting to academic oral surgery posts, succession planning, clinical service need and financial challenges facing Universities that may all influence patient safety in the educational setting. 
This survey explored UK dental schools' undergraduate experience of WSS, the changes implemented and their impact on undergraduate student experience. Limitations of the survey include the inability to capture accessible and accurate data on NEs prior to the introduction of electronic incident reporting systems within these Trusts. The implementation of these systems would have occurred over a variable time period across the UK. Furthermore, parts of the survey rely on individual teaching staff's opinions, despite this however, the responses are representative of all schools in the UK and have identified distinctly common themes. The significant missing part of this survey is the voice of the student, their opinion of patient safety changes introduced and the impact this has had on learning. Further studies are needed to investigate this important element. 


\section{Conclusion}

Our survey highlights that all UK Dental Schools are embracing the national challenge of increased emphasis on patient safety against the backdrop of a unique undergraduate course where students undertake surgery with the potential for harm. Almost all schools have introduced patient safety measures, thus modelling a patient safety mindset to students, and increased staff supervision.

The Dental Schools have shown openness in sharing data on the incidence of undergraduate WSS as well as a willingness to learn from these errors. The impact of this increasing patient safety focus on student's clinical experience is reported to be mixed, according to respondents of this survey. Increased student awareness of patient safety is acknowledged as a significant benefit. In particular, the perception of better patient experience and higher quality teaching associated with increased supervision offset the negatives of less experience and lack of autonomous practice. A proposed safety standard is suggested to ensure a balance can be achieved between effective patient safety measures and adequate staffing, ensuring students gain sufficient experience to be able to work autonomously upon qualification. After all, sending safe, competent and confident dental practitioners into dental practice is the 'raison d'etre' of all dental schools.

\section{Conflict of Interest}

The authors certify that they have no conflict of interest.

\section{Data Availability Statement}

The data that support the findings of this study are available from the corresponding author upon reasonable request. 


\section{References}

1. To Err is Human: Building a safer health system. Washington Metropolitan Area, Institute of Medicine, 1999. https://www.nap.edu/resource/9728/To-Err-is-Human1999--report-brief.pdf Accessed: 10/06/2020

2. Report of the Mid Staffordshire NHS Foundation Trust Public Inquiry, chaired by Robert Francis QC. 2013.

https://assets.publishing.service.gov.uk/government/uploads/system/uploads/attac hment data/file/279124/0947.pdf Accessed: 10/06/2020

3. A promise to learn - a commitment to act. Improving the safety of patients in England. National Advisory Group on the Safety of Patients in England. 2013. https://assets.publishing.service.gov.uk/government/uploads/system/uploads/attac hment data/file/226703/Berwick Report.pdf Accessed: 10/06/2020

4. Preparing for practice, General Dental Council 2015. https://www.gdcuk.org/docs/defaul;t-source/quality-assurance/preparing-for-practice-(revised2015).pdf Accessed: 11/06/2020

5. Standards for Education, General Dental Council 2015. https://www.gdcuk.org/docs/default-source/quality-assurance/standards-for-education-(revised2015).pdf?sfvrsn=1f1a3f8a 2 Accessed 11/06/2020

6. Field JC, Cowpe JG, Walmsley AD. The Graduating European Dentist: A New Undergraduate Curriculum Framework. Eur J Dent Educ. 2017; 21 (Suppl. 1): 2-10. https://doi.org/10.1111/eje.12307

7. Wright S, Crofts G, Ucer C, Speechley D. Errors and Adverse Events in Dentistry - A Review. Dental Update 2017; 44: 979-982.

8. Leong P, Afrow J, Weber HP, Howell H. Attitudes Toward Patient Safety Standards in US Dental Schools: A Pilot Study. J Dent Educ. 2008; 72 (4): 431-437.

9. Palmer JC, Blanchard JR, Jones J, Bailey E. Attitudes of Dental Undergraduate Students Towards Patient Safety in a UK Dental School. Eur J Dent Educ. 2019; 23: 127-134.

10. Never events policy and framework, revised January 2018. https://improvement.nhs.uk/documents/2265/Revised_Never_Events policy and f ramework FINAL.pdf Accessed: 10/06/2020

11. Revised never events policy and framework, March 2015. https://www.england.nhs.uk/wp-content/uploads/2015/04/never-evnts-polframwrk-apr.pdf Accessed: 11/06/2020 
12. Never events data, NHS Improvement. https://improvement.nhs.uk/resources/never-events-data/ Accessed: 11/06/2020

13. Pemberton MN. Wrong tooth extraction: further analysis of "never event" data. $\mathrm{Br} J$ Oral Maxillofac Surg. 2019; 57: 932-934.

14. Chang H-H, Lee J-J, Cheng S-J, Yang P-J, Hahn L-J, Kuo Y-S, Lan W-H, Kok S-H. Effectiveness of an Educational Program in Reducing the Incidence of Wrong-site Tooth Extraction. Oral Surg Oral Med Oral Pathol Oral Radiol Endod. 2004; 98: 28894.

15. Peleg O, Givot N, Halamish-Shani T, Taicher S. Wrong Tooth Extraction: Root cause analysis. Quintessence Intl. 2010; 41 (10): 869.72.

16. Singh $\mathrm{H}$, Thomas EJ, Peterson LA, Studdert DM. Medical errors involving trainees: a study of closed malpractice claims from five insurers. Arch Intern Med. 2007; 167: (19) 2030-6.

17. Sorokin R, Riggio JM, Hwang C. Attitudes about patient safety: a survey of physiciansin-training. Am J Med Qual. 2005; 20: (2) 70-7.

18. Pemberton MN. Surgical Safety Checklists and Understanding of Never Events, in UK and Irish Dental Hospitals. Br Dent J. 2016; 220: 585-589.

19. Atkin PA, Willis A, Doncahie C, Elledge ROC, Thomas SJ, Riordain RN et al. Human disease/clinical medical sciences in dentistry: Current state and future development of undergraduate assessments in the UK and Ireland. Eur J Dent Educ. 2020; 24: 442448.

20. Saksena A, Pemberton MN, Shaw A, Dickson S, Ashley MP. Preventing wrong tooth extraction: experience in development and implementation of an outpatient safety checklist. Br Dent J. 2014; 217: (7) 357-362.

21. Fyfe $\mathrm{E}$, Fleming $\mathrm{C}$. The WHO surgical safety checklist in a dental-teaching hospital department of oral surgery - a model for implementation. Oral Surgery. 2013; 6: 180-185.

22. Bailey E, Tickle M, Campbell S, O'Malley L. Systematic review of patient safety interventions in dentistry. BMC Oral Health. 2015; 15: 152.

23. NHS England. Standardise, educate, harmonise. Commissioning the conditions for safer surgery. Report of the NHS England Never Events Taskforce, February 2014. https://improvement.nhs.uk/documents/922/sur-nev-ev-tf.pdf Accessed: $23 / 06 / 2020$

24. Graham C, Reid S, Lord TC, Taylor KH. The evolution of patient safety procedures in an oral surgery department. Br Dent J. 2019; 226 (1) 32-38. 
25. Raja S, Rajagopalan CF, Patel J, Van Kanegan K. Teaching dental students about patient communication following an adverse event: a pilot educational module. $J$ Dent Educ. 2014; 78: (5) 757-762.

26. Macluskey $M$ and Durham J. Oral surgery undergraduate teaching and experience in the United Kingdom: a national survey. Eur J Dent Ed. 2009; 13: 52-57.

27. Schumacher DJ, Slovin SR, Riebschleger MP et al. Beyond counting hours: the importance of supervision professionalism transitions of care and workload in residency training. Acad Med. 2012; 87: 883-8.

28. Ali K, Tredwin C, Kay E, Slade A. Transition of new dental graduates into practice: a qualitative study. Eur J Dent Educ. 2016; 20: 65-72.

29. Leadbetter D, Madden J, Ross B, Russell E. Transition to dental practice: Newly graduated dentists' views of being successful in dental practice. Eur J Dent Educ. 2020; 24: 753-762.

30. Shah S, Halai T, Patel J, Sproat C. Perceived confidence and experience in oral surgery among final year undergraduate students in a UK dental school. Br Dent J. 2018; 224: 177-182.

31. Macluskey M, Durham J, Bell A, Cowpe J, Crean St. J, Dargue A et al. A national survey of UK final year students' opinion of undergraduate oral surgery teaching. Eur J Dent Educ. 2011; 16: e205-e212.

32. Bloom BS. Mastery learning. Evaluation comment. 1968; 1: (2) University of California, LA, Center for the Study of Evaluation of Instructional Programs. https://programs.honolulu.hawaii.edu/intranet/sites/programs.honolulu.hawaii.edu .intranet/files/upstf-student-success-bloom-1968.pdf Accessed: 21/10/2020

33. Subramanian J, Anderson VR, Morgaine KC, Thomson WM. Effective and ineffective supervision in postgraduate dental education: a qualitative study. Eur J Dent Educ. 2012; 17: e142-e150. 


\section{Table 1}

Numbers of Wrong Tooth/Teeth Extracted and "Never Events" reported as occurring between 2015 - 2020 during NHS care in England

\begin{tabular}{|l|l|c|c|c|}
\hline Year Period & $\begin{array}{l}\text { Total Number } \\
\text { of Wrong } \\
\text { Tooth/Teeth } \\
\text { Extracted } \\
\text { reported to } \\
\text { NHS England }\end{array}$ & $\begin{array}{l}\text { Total Number } \\
\text { of all WSSs } \\
\text { reported to } \\
\text { NHS England }\end{array}$ & $\begin{array}{l}\text { Total Number of } \\
\text { NEs reported to } \\
\text { NHS England }\end{array}$ & $\begin{array}{l}\text { Wrong } \\
\text { Tooth/Teeth } \\
\text { Extraction as } \\
\text { Percentage of } \\
\text { Total NEs } \\
\text { Reported }\end{array}$ \\
\hline $2015-2016$ & 33 & 179 & 442 & $7 \%$ \\
\hline $2016-2017$ & 46 & 189 & 445 & $10 \%$ \\
\hline $2017-2018$ & 28 & 175 & 407 & $7 \%$ \\
\hline $2018-2019+$ & 42 & 207 & 496 & $8 \%$ \\
\hline $2019-2020+$ & 38 & 218 & 435 & $9 \%$ \\
\hline
\end{tabular}

${ }^{+}$Provisional publication data 


\section{Figure 1}

Levels of Dental Student Supervision whilst Undertaking Routine Extractions in UK Dental Schools in 2019

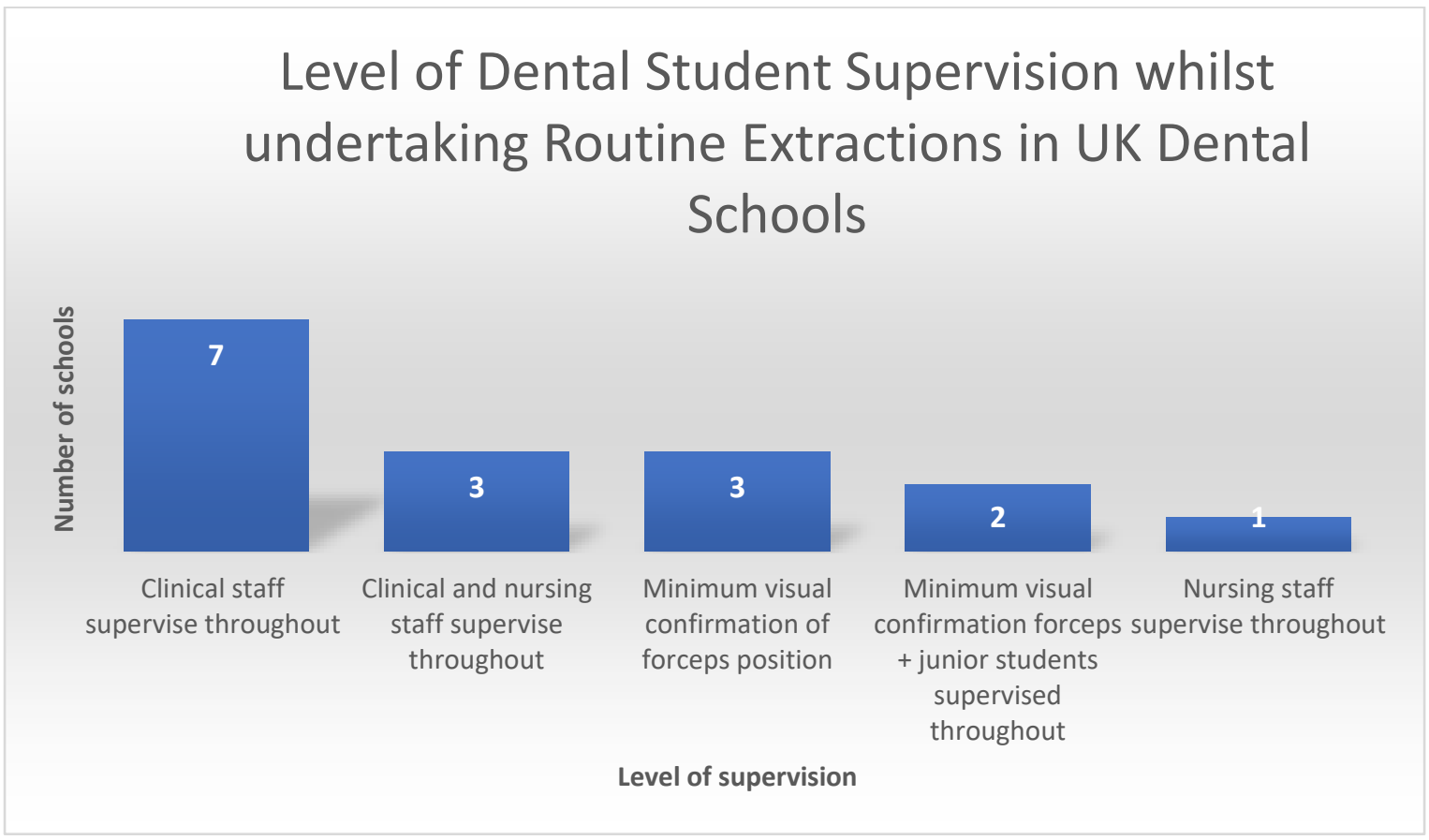




\section{Table 2}

Levels of Staff Supervision of Undergraduate Dental Students Performing Extractions in relation to the Average Number of Students per Year

\begin{tabular}{|c|c|c|}
\hline $\begin{array}{c}\text { Average number of dental } \\
\text { students per year }\end{array}$ & $\begin{array}{c}\text { Ratio of staff: student } \\
\text { supervision }\end{array}$ & $\begin{array}{c}\text { Number of dental schools } \\
\text { (\% schools) }\end{array}$ \\
\hline Less than 40 & $1: 1$ & $1(6 \%)$ \\
\hline $60-80$ & $1: 3$ & $5(31 \%)$ \\
\hline $60-80$ & $1: 4$ & $5(31 \%)$ \\
\hline $80-100$ & $1: 4$ & $3(19 \%)$ \\
\hline $80-100$ & $1: 6$ & $1(6 \%)$ \\
\hline $140+$ & $1: 3$ & $1(6 \%)$ \\
\hline
\end{tabular}


Figure 2

Total Number of Wrong Tooth Extractions (NE's) by Undergraduates in UK Dental Schools 2009 - 2019

Number of Wrong Tooth Extractions (NE) in Dental Teaching Hospitals 2009 - 2019

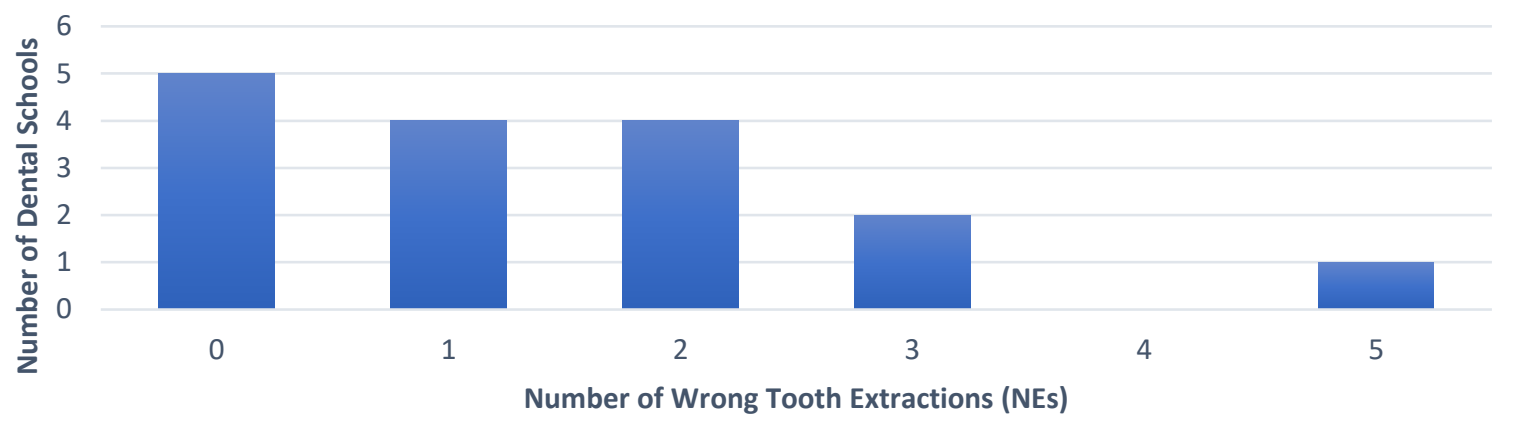




\section{Table 3}

Agreement with Statements about How Interventions to Reduce WSS have Impacted on Student Experience

\begin{tabular}{|l|c|}
\hline Statement - Positive Effect & $\begin{array}{c}\text { Frequency of agreement } \\
\text { (\% schools) }\end{array}$ \\
\hline Formalised process for checks & $13(81 \%)$ \\
\hline Students more mindful/aware of patient safety & $9(56 \%)$ \\
\hline Patient experience improved & $4(31 \%)$ \\
\hline Higher quality teaching & $3(19 \%)$ \\
\hline Standardised teaching & $10(63 \%)$ \\
\hline Statement - Negative effect & $10(63 \%)$ \\
\hline Students get less opportunity to work & $8(50 \%)$ \\
\hline independently & $8(50 \%)$ \\
\hline More paperwork & $6(38 \%)$ \\
\hline Students see fewer patients per session & $2(13 \%)$ \\
\hline Longer waiting time for supervisor & $3(19 \%)$ \\
\hline Students don't understand significance of & \\
\hline safety checks & \\
\hline Minimal effect on patient safety & \\
\hline Creates blame culture & \\
\hline
\end{tabular}




\section{Figure 3}

Proposed Minimum Safety Standard for Extractions by Undergraduate Dental Students

1. Pre-operative safety briefing with student group

2. Patient treatment

Whiteboard used to record teeth for extraction at that visit

Modified WHO checklist

Direct clinical staff supervision, with discretion for senior students

Minimum ratio of 1 staff member to 3 students undertaking treatment 


\section{Appendix 1}

Undergraduate Never Event Questionnaire

Q1. What is the name of your teaching hospital?

Q2. When did you introduce a WHO checklist (correct site surgery) into your undergraduate extraction sessions?

$2009-2014$

$2015-2019$

Not introduced If not, can you give more detail on why?

Q3. Do you use a pre-operative safety briefing with undergraduate students prior to a session where they perform extractions?

Yes

No

Other

Q4. What other pre-operative precautions/checks do you undertake prior to the student beginning an extraction case, apart from the WHO checklist?

Tooth/site marking

Bib marking

Board marking

Other, please specify

Q5. Please tick what kind of staff supervision students have, when undertaking routine extractions?

Clinical staff supervise all student years throughout

Nursing staff supervise all student years throughout

Supervise only junior students throughout

Only visually confirm forceps position

No direct supervision

Q6. Have any of your undergraduate students taken out the wrong tooth since 2009?

If yes, please specify how many times:

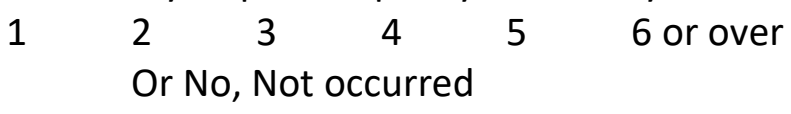

Q7. What changes have been implemented since these events?

Tooth/site marking

Bib marking

Board marking

WHO safety checklist

Pre-op safety briefing

Direct clinical staff supervision

Direct nursing staff supervision

Buddy/partnering of students

No changes made because no NE's 
Any other comments

Q8. What kind of effect do you think the changes implemented following NE's and increased patient safety have had on the undergraduate dental student's experience, and why?

Positive

Negative

Mixed

No effect

Don't know

Q9. Please can you mark your agreement with any of the following statements in terms of the impact of changes implemented following never events on the undergraduate's experience of extractions:

Standardised teaching

Formalised process for checks

Higher quality teaching

Students more mindful/aware

Patient experience improved

Students get less opportunity to work independently

Students don't understand significance of safety checks

More paperwork

Students see fewer patients/session

Longer waiting time for supervisor

Creates blame culture

Minimal effect on patient safety

Q10. On average how many dental students in each clinical year?

$\begin{array}{llllll}\text { Less than } 40 & 60-80 & 80-100 & 100-120 & 120-140 & 140+\end{array}$

Q11. On average what is the ratio of Staff: Students when extracting teeth?

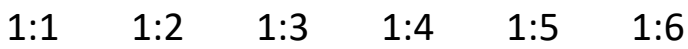

